\title{
Angiographically minimal but functionally significant coronary lesion confirmed by optical coherence tomography
}

Hyuck-Jun Yoon, Yun-Kyeong Cho, Chang-Wook Nam, Kwon-Bae Kim, and Seung-Ho Hur

Department of Internal Medicine, Keimyung University Dongsan Medical Center, Daegu, Korea

Received: March 13, 2015

Revised : March 23, 2015

Accepted: April 22, 2015

\section{Correspondence to}

Seung-Ho Hur, M.D.

Tel: +82-53-250-7998

Fax: +82-53-250-7034

E-mail:shur@dsmc.or.kr
A 48-year-old man visited our hospital due to exertional chest pain. His resting electrocardiogram exhibited a $\mathrm{T}$ wave inversion in the inferior leads. A transthoracic echocardiogram showed a preserved left ventricular systolic function without any regional wall motion abnormalities. Multi-detector computed tomography was performed to evaluate

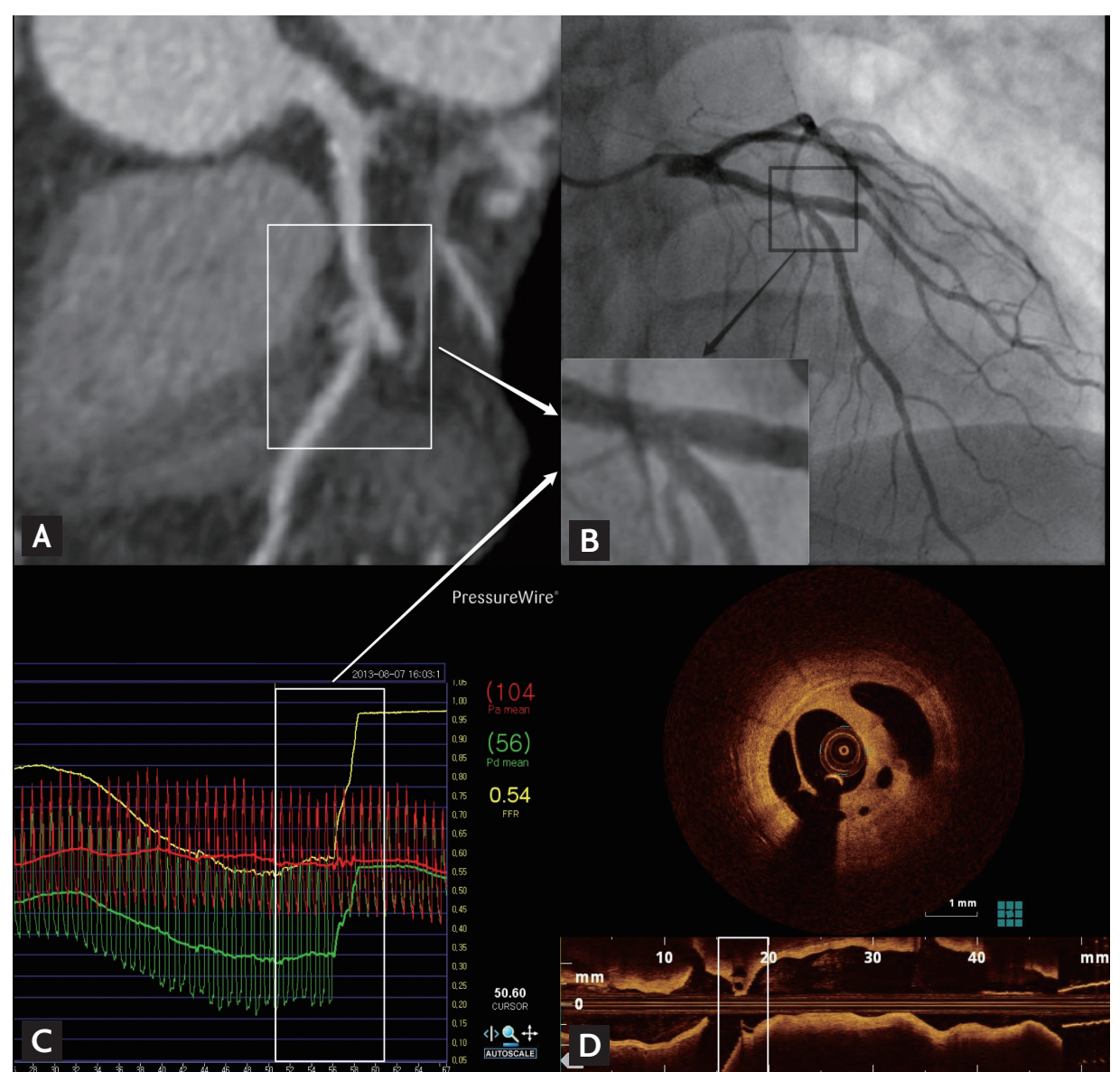

the existence of any coronary artery disease and it revealed a significant stenosis in the right coronary artery; however, there was a minimal atherosclerotic lesion in the left coronary artery (Fig. 1A). The right coronary angiogram showed a significant stenosis in the mid portion of the right coronary artery and percutaneous coronary intervention for that
Figure 1. (A) Multi-detector computed tomography shows a minimal coronary stenosis in the left anterior descending artery (LAD). (B) The coronary angiogram also shows no significant coronary lesions in the LAD. (C) The fractional flow reserve (FFR) measurement using a pressure wire showed a significant functional defect (FFR, 0.54) in the LAD and steep step up of the FFR value at the bifurcation of the LAD and first diagonal branch. (D) Optical coherence tomography revealed a focal (2 $\mathrm{mm}$ length) lotus root-like lesion consisting of multiple cavities divided by septa within mild fibrous plaque. In spite of the variability in the size of the channel, microchannels were seen to communicate with each other. 
lesion was performed successfully with a drug-eluting stent. The left coronary angiogram showed a focal minimal lesion in the mid left anterior descending artery, just proximal to the diagonal bifurcation (Fig. 1B). Contrary to our expectations, the fractional flow reserve (FFR) measured by a pressure wire at that lesion was significantly decreased at 0.54 (Fig. 1C). Intravascular optical coherence tomography (OCT) was performed to evaluate the cause of the discordance between the angiography and FFR. The OCT revealed a focal ( $2 \mathrm{~mm}$ length) lotus root-like lesion consisting of multiple cavities divided by septa within fibrous plaque (Fig. 1D). In spite of the variability in the size of the channels, microchannels were seen to communicate with each other. That was a suspicious finding of a recanalized intracoronary thrombus. That lesion was also successfully revascularized and the patient's symp- toms no longer occurred after discharge.

Decision making for revascularization based on angiography alone without prior vessel specific stress testing can be difficult in patients with multi-vessel coronary disease including intermediate or ambiguous coronary lesions. Therefore, functional and physiological considerations of such coronary lesions should be warranted for the selection of the target vessel. This representative case well demonstrated a noteworthy risk of an underestimation using coronary angiography, which could be compensated by invasive functional or intravascular imaging procedures.

\section{Conflict of interest}

No potential conflict of interest relevant to this article was reported. 